\title{
Sexual Abuse among Female Undergraduates in Tertiary Institutions in IMO State, Southeast Nigeria: Prevalence, Pattern and Determinants
}

\author{
Chukwuma B. Duru1 ${ }^{*}$, Chinyere M. Aguocha ${ }^{2}$, Anthony C. Iwu ${ }^{1}$, Uche R. Oluoha ${ }^{1}$, \\ Ijeoma N. Okedo-Alex ${ }^{3}$, Ikechi Ohanle ${ }^{4}$, Ernest Nwaigbo ${ }^{4}$ \\ ${ }^{1}$ Department of Community Medicine, Imo State University, Owerri, Nigeria \\ ${ }^{2}$ Department of Psychiatry, Imo State University, Owerri, Nigeria \\ ${ }^{3}$ Department of Community Medicine, Federal Teaching Hospital, Abakaliki, Nigeria \\ ${ }^{4}$ Department of Community Medicine, Imo State University Teaching Hospital, Orlu, Nigeria \\ Email:^duruchukwuma16@yahoo.com,*drduruchukwuma@gmail.com
}

How to cite this paper: Duru, C.B., Aguocha, C.M., Iwu, A.C., Oluoha, U.R., Okedo-Alex, I.N., Ohanle, I. and Nwaigbo, E. (2018) Sexual Abuse among Female Undergraduates in Tertiary Institutions in IMO State, Southeast Nigeria: Prevalence, Pattern and Determinants. Open Access Library Journal, 5: e4516.

https://doi.org/10.4236/oalib.1104516

Received: March 19, 2018

Accepted: April 10, 2018

Published: April 13, 2018

Copyright $\odot 2018$ by authors and Open Access Library Inc.

This work is licensed under the Creative Commons Attribution International License (CC BY 4.0).

http://creativecommons.org/licenses/by/4.0/

\section{(c) () Open Access}

\begin{abstract}
Introduction: Sexual abuse is a global public health problem that cuts across social class, cultures, tribes etc. and has permeated the fabrics of tertiary institutions and many work places as long as humans have reasons to interact. Aim: To determine the prevalence, pattern and determinants of sexual abuse among undergraduates in tertiary institutions in Imo State, Nigeria. Methodology: This study was a descriptive, cross sectional survey of 600 female students in tertiary institutions in Imo State Nigeria. They were selected using the multistage sampling technique and data were collected using a pretested, semi-structural and self-administered questionnaire and analyzed using a computer soft-ware (EPI INFO version 3.3.2). Descriptive statistics was presented using summary indices, frequency tables and percentages. Associations between variables were done using the chi-square and logistic regression. A p-value of less than 0.05 was considered significant. Results: The mean age of the respondents was $21.7+3.2$ years. The current and life-time prevalence of sexual abuse among the respondents was $15.2 \%$ and $34 \%$ respectively and the commonest form of sexual abuse experienced was founding/grabbing of sensitive body parts (52.5\%). Factors found to influence current occurrence of sexual abuse among the students were; age $(p=0.010)$, tribe $(p=0.008)$, marital status $(\mathrm{p}<0.001)$, type of student in secondary school attended $(\mathrm{p}=$ $0.001)$, faculty of study $(\mathrm{p}=0)$, year in school $(\mathrm{p}<0.0001)$, persons currently shared room with $(\mathrm{p}<0.0001)$ and level of knowledge about sexual abuse $(\mathrm{p}=$ 0.007). Conclusion: The prevalence of abuse as revealed in this study was high. There is need to develop appropriated prevention strategies by the universities to curb this menace against our women.
\end{abstract}




\section{Subject Areas}

Education, Sports Science

\section{Keywords}

Sexual Abuse, Prevalence, Pattern, Determinants, Female, Tertiary

Institutions, Imo State

\section{Introduction}

Gender-based violence against women, which is a public health problem, has been acknowledged worldwide as a violation of basic human rights. An increasing amount of research highlights the health burdens, intergenerational effects, and demographic consequences of such violence [1] [2]. The World Health Organization defines such violence as "the intentional use of physical force or power, threatened or actual, against oneself, another person, or against a group or community, that either results in or has a high likelihood of resulting in injury, death, psychological harm, or deprivation" [3]. Sexual abuse which is a form of such violence also referred to as molestation, is the forcing of undesired sexual behavior by one person upon another. When that force is immediate, of short duration, or infrequent, it is called sexual assault [4]. American Psychological Association (APA) defined sexual abuse as an unwanted sexual activity with perpetrators using force, making threats or taking advantage of victims notable to give consent [5]. Sexual abuse may take many forms and vary in terms of frequency, duration, invasiveness of the acts involved, and the use of force or coercion [6]. The term sexual abuse also covers any behavior by an adult towards a child to stimulate either the adult or the child sexually [7]. Both sexes are affected, though most of the cases occur among females, especially female children and adolescents. Contrary to myth that most perpetrators are strangers, wide spread research has revealed that most of the assailants are usually intimate partners, friends and someone within the family. Males perpetrate the majority (80\% - 95\%) of sexual abuse, though there are certainly some cases in which female offenders victimize male or female children [5] [8] [9] [10] [11]. Sexual abuse or harassment is a global issue that cuts across, social class, cultures, tribes etc., and has permeated the fabrics of tertiary institutions, and many work places as long as human have reasons to interact [12] [13]. The actual prevalence of sexual abuse/violence is unknown as a result of gross under-reporting even though it has been reported as the fastest growing crime in many parts of the world [14] [15]. However, in a WHO Multi-country survey, the life-time prevalence of sexual partner violence reported by women, aged $15-49$ years range from $6 \%$ in Japan to $59 \%$ in Ethiopia, with rates in the majority of cases falling between $10 \%$ and $50 \%$ [16] [17]. Studies have shown that 1 in 4 women may have experienced sexual violence by an intimate partner and up to a third of 
adolescent girls reported their experience as being forced [18] [19] [20]. Almost 1 in 5 women has been sexually abused by a man at some time in her life [14]. In a 2011 survey of high students in US, $11.8 \%$ of girls and $4.5 \%$ of boys from grades 9 - 12 reported that they were forced to have sexual intercourse at some time in their lives [21], while a study of undergraduate women also in the US revealed that $19 \%$ of them experienced attempted or completed sexual assault since entering college [22]. In Africa the prevalence was generally high and varies between countries; it was $16 \%$ in Cameroun, $23 \%$ in Sierra Leone, $34.4 \%$ in Ethiopia, 49\% in Ghana, 65.6\% in Zimbabwe and 67\% in Botswana [23]-[28]. In Nigeria, reports also vary from the different regions of the country. A study from a university in the South eastern part of the country reported 36.7\% [29], studies from three South-south universities reported; 51.7\% [30], 46.7\% [31] and 18.4\% [32] respectively while lower figures were reported from two institutions in Northern Nigeria (13.8\% and 22.2\%) [33] [34]. Some of the predisposing factors and determinants of sexual abuse/violence reported in previous studies were; age, sex, marital status of female, socioeconomic status of women and poverty, educational status of women, occupation, living in arrangement, orphan status, type of family, current parents, marital status, alcohol consumption, drug use, previous experience of rape or sexual abuse, multiple sexual partners, younger age at coitarche, history of forced coitarche, coitarche with relatives and unknown persons, year of study and living status of student and being a college or university student among others [29] [31] [33]-[41]. Studies have shown that sexual abuse/violence have negative impacts on the physical, social and mental health of the victim [31] [36] [41]. Some of the consequences include reproductive health problems like unwanted pregnancy, sexually transmitted diseases/HIV etc., all forms of injuries, depression, anxiety, social isolation, loss of self-esteem, distrust of others, substance abuse, post-traumatic disorder, disability and even death [31] [42] [43] [44]. The problem of sexual abuse against female undergraduates in our tertiary institutions has remained largely unaddressed, it prevents this students from attaining their maximum intellectual, emotional and educational potentials [31]. It is a psychological threat to a conducive learning environment [30] [31]. Various forms of sexual abuse has been reported to occur among undergraduates in university setting which include rape (attempted or completed), unwanted sexual advances, sexual harassment, demanding sex in-return for favor, sexual abuse of mentally or physically disabled people, pornography and internet sexual abuse, fondling and grabbing of sensitive body parts among others [30] [31]. Despite the problems caused by sexual violence in our environment, majority of the cases were not reported, primarily because of cultural issues and patrichial nature of our society with male dominance [30] [31] [40]. This has led to rising cases of abuse especially in our universities, where even reported cases has not been give appropriate attention and the culprit move freely thereby worsening the state of the abused females. 
Therefore our study aim is to assess the prevalence, pattern and determinants of sexual abuse among females in tertiary institutions in Imo State with a view of informing policy makers and the public of the burden of sexual abuse and how this can be greatly reduced and save our women from its long term effects. Theses study findings will help to reveal the magnitude of sexual abuse, the prevalent patterns of sexual abuse and the main perpetrators of this senseless act in our tertiary institutions in Imo state, Nigeria. Also the short and long term health and mental consequences of sexual abuse will be revealed which will in turn guide our policy makers in developing appropriate policies that will help curb this menace in our institutions of higher learning.

\section{Methodology}

\subsection{Study Area}

Imo State is one of the 36 States of Nigeria in the South Eastern Region of the country. It has a total population of about 3.93 million people, comprising more males than females (2.03 million and 1.9 million people respectively) and an annual growth rate of about $3.0 \%$. The majority of the people living in the State are of Igbo tribe extraction and the state has one the highest literacy rate in the country of over $95 \%$. Important educational institutions in the state include; Imo State University, Federal University of Technology, Eastern Palm University, Alvan Ikoku Federal College of Education, Federal Polytechnic, Nekede, Imo State Polytechnic, School of Nursing and Health Sciences, African Institute of Science and Technology, Seat of Wisdom Seminary School among others.

\subsection{Study Population and Design}

The study population comprised female undergraduate students of the selected institutions in Owerri Imo state. This is a descriptive cross sectional study on the prevalence, pattern and determinants of sexual abuse among female students in tertiary institutions in Imo State, South East, Nigeria.

\subsection{Inclusion and Exclusion Criteria}

1) Inclusion: Only female students of the selected institutions were studied whether they were on part time or full-time basis were enrolled and studied

2) Exclusion criteria: All visitors to the institution were excluded no matter their status. All male students, lecturers and staff of the institution were excluded for the survey.

\subsection{Sample Size Determination}

Using the Cochran sample size formula for cross sectional studies in populations greater than 10,000 people and the prevalence of sexual abuse in a closely related study from Nigeria, with a prevalence of $46.7 \%$ [31], and applying the formula below, the minimum sample size required for the study was 366 students.

$$
n=Z^{2} P q / d^{245}
$$


where $n$ is the minimum sample size, $Z=$ standard normal deviate set at $95 \%$ significance level $\approx 1.96, p=$ the prevalence of life-time abuse from a previous study $=46.7 \%, q=1-p, d=$ the degree of accuracy set at 0.05 . But a sample size of 600 students were enrolled and studied to cover for attrition.

\subsection{Sampling Technique}

The study was carried out using the multistage sampling technique.

STAGE 1: This involved the stratification of the institutions into two categories; category A comprised Universities while category B comprised other higher institutions excluding universities and using simple random sampling by balloting one institution was selected from each category. Three Hundred students each was studied in both institutions.

STAGE 11: This involved selection of the Faculties and Departments to be studied. This was done using proportionate simple random sampling by balloting based on population size of the selected Faculties and Departments.

STAGE 111: This involves the selection of study participants. This was done using cluster sampling technique. The classes studied were grouped into clusters from 100 - 600 level. And one cluster from each class was selected using simple random sampling. All selected clusters were studied.

\subsection{Data Collection and Analysis}

Data was collected using a pretested, semi-structured, self-administered questionnaire. The questionnaire contained three sections; Section A: Socio demographic characteristics of respondents, Section B: Awareness and knowledge about sexual abuse, Section C: Prevalence and experience of sexual abuse among students. Quantitative data collected were cleaned and validated manually, while a computer software package (EPI INFO version 3.3.2) was used for data entry and analysis. Frequencies and percentages of relevant variables were generated, bivariate analysis conducted using Chi-square and logistic regression to test associations between variables. For the purpose of this study, knowledge of respondents about sexual abuse was scored using five awareness questions, each question assigned scores 0 - 5 with a maximum total score of 25 for all the five questions. The score was graded as poor if the respondent scored $0 \%-49 \%$ of the total, fair if the respondent scored 50\% - 69\% of the total and high/good knowledge if the respondents scored $\geq 70 \%$ of the total score. A p-value $<0.05$ was considered statistically significant.

\subsection{Ethical Approval}

Ethical approval was gotten from the Faculty of Clinical Medicine, Madonna University Nigeria, before proceeding to the study. Approvals were gotten from the relevant university authorities and verbal consent was obtained from all participants after full explanation of the purpose of the study before proceeding to

the interview. The research was conducted in line with laid down procedures at 
Helsinki (1964) in use of human subjects.

\section{Result}

\subsection{Socio-Demographic Characteristics of Respondents}

The mean age of respondents was $21.7 \pm 3.2$ years and the majority of the undergraduates studied were singles (87\%). A higher proportion of them attended private secondary schools $241(40.2 \%)$, that were mainly situated in the urban areas (66.7\%). Majority of them attended mixed schools 356 (59.3\%), were day students only, (60.7\%) and were in the faculty of Arts/Education, (61.7\%). Most of the students were above 100 level, (93.5\%), of which majority of them reside off campus (53.5\%), share room with someone (80.5\%) and many of those who share room with someone do that with their female friends 295 (61.1\%). Most of the respondents were sponsored in school by family members $(95.8 \%)$ and were of Igbo extraction, (92.5\%). A higher proportion of them were Catholics (48.7\%) (Table 1 and Table 2).

\subsection{Awareness and Knowledge about Domestic Violence}

Most of the respondents, (81.8\%) have heard about sexual abuse and the common sources of information were from; television, (61.1\%), newspaper, (53.8\%), friends, (53.0\%), and radio (51.9\%). The most common definition of sexual abuse known was sexual act without consent (89.2\%), and common forms of sexual abuse mentioned were; Fondling/grabbing of private body parts (86.4\%), rape $(66.8 \%)$ and exposure to pornography $(46.4 \%)$. Common victims of sexual abuse mentioned were; children, (74.1\%), females, (69.0\%) and singles, (57.2\%). Majority of the respondents (96.3\%) mentioned females as the genders most affected. Teacher/lecturers (72.3\%), intimate partners (44.2\%) were the common perpetrators of abuse mentioned. Commonest place that abuse takes place was in secretes corners $(71.1 \%)$, followed closely by school/office $(67.4 \%)$ and clubs (58.7\%). Transmission of STI (77.8\%) was the commonest consequence of abuse mentioned. A higher proportion of the students had poor knowledge about sexual abuse, (47.0\%). The commonest factor leading to sexual abuse suggested by respondents was ignorance, $214(14.4 \%)$ followed by financial problem, 196 (13.2\%) and indecent dressing, 158 (10.6\%). The commonest preventive measures to stop sexual abuse suggested by respondents were self-control 336 (17.3\%) followed by stopping of lonely visits 225 (11.6\%), punishment for offenders 215 (11.1\%), and avoidance of bad company 185 (9.5\%) (Table 3).

\subsection{Prevalence and Pattern of Sexual Abuse}

About one-third (34\%) of the students had experienced sexual abuse in their life-time with majority of them, (54.9\%) experiencing it more than once in their life-time. The major forms of sexual abuse ever experienced were; fondling/grabbing of sensitive body parts/kissing, (52.5\%), rape, (19.1\%) and sex for favor, (13.2\%). The commonest scene of occurrence of life-time sexual abuse 
Table 1. Sociodermographic characteristics of respondents.

\begin{tabular}{|c|c|c|}
\hline VARIABLE & FREQUENCY & PERCENTAGE \\
\hline \multicolumn{3}{|l|}{ AGE GROUP } \\
\hline$<20$ & 230 & 38.3 \\
\hline $21-25$ & 306 & 51.0 \\
\hline$>26$ & 64 & 9.7 \\
\hline Total & 600 & 100 \\
\hline \multicolumn{3}{|l|}{ MARITAL STATUS } \\
\hline Single & 522 & 87.0 \\
\hline Ever married & 78 & 13.0 \\
\hline Total & 600 & 100 \\
\hline \multicolumn{3}{|c|}{ TYPE OF SECONDARY SCHOOL ATTENDED } \\
\hline Public & 239 & 39.8 \\
\hline Private & 241 & 40.2 \\
\hline Missions & 120 & 20.0 \\
\hline Total & 600 & 100 \\
\hline \multicolumn{3}{|c|}{ LOCATION OF SECONDARY SCHOOL } \\
\hline ATTENDED & & \\
\hline Urban & 400 & $\begin{array}{l}66.7 \\
333\end{array}$ \\
\hline Rural & 200 & 33.3 \\
\hline Total & 600 & 100 \\
\hline \multicolumn{3}{|c|}{ FORM OF SECONDARY SCHOOL ATTENDED } \\
\hline Girls only & 244 & 40.7 \\
\hline Mixed school & 356 & 59.3 \\
\hline Total & 600 & 100 \\
\hline \multicolumn{3}{|c|}{ TYPE OF STUDENT IN SECONDARY SCHOOL } \\
\hline Boarding student only & 176 & 29.3 \\
\hline Day student only & 364 & 60.7 \\
\hline Both & 60 & 10.0 \\
\hline Total & 600 & 100 \\
\hline \multicolumn{3}{|l|}{ FACULTY OF STUDY } \\
\hline Art/Education & 370 & 61.7 \\
\hline Social sciences/Sciences & 150 & 25.0 \\
\hline Health sciences & 80 & 13.3 \\
\hline Total & 600 & 100 \\
\hline \multicolumn{3}{|l|}{ LEVEL } \\
\hline 100 & 39 & 6.5 \\
\hline 200 & 118 & 19.7 \\
\hline 300 & 246 & 41.0 \\
\hline$>400$ & 197 & 32.9 \\
\hline Total & 600 & 100 \\
\hline \multicolumn{3}{|c|}{ RESIDENCE IN CURRENT SCHOOL } \\
\hline In-school hostel & 155 & 25.8 \\
\hline Off campus hostel & 321 & 53.5 \\
\hline Family house & 124 & 20.7 \\
\hline Total & 600 & 100 \\
\hline \multicolumn{3}{|c|}{ SHARING ROOM WITH SOMEONE } \\
\hline Yes & 483 & 80.5 \\
\hline No & 117 & 19.5 \\
\hline Total & 600 & 100 \\
\hline
\end{tabular}




\section{Continued}

PERSON SHARED ROOM WITH $(n=483)$

Female friend
Boyfriend
Relative
Total

SPONSORSHIP IN SCHOOL

Non-self
Self
Total

$\begin{array}{cc}575 & 95.8 \\ 25 & 4.2 \\ 600 & 100\end{array}$

\section{RELIGION}

Catholic

Pentecostal

Orthodox

ISLAM

Total

TRIBE

reported was home of perpetuators, $(72.6 \%)$. The common circumstances that lead to the abuse were; visiting the house of the perpetuator, $(62.7 \%)$ and violence/force, (34.8\%). Current experience of sexual abuse among the students was $15.2 \%$ and the commonest form of sexual abuse experienced was fondling/grabbing of sensitive body parts/kissing, (59.3\%) followed by rape, $(40.7 \%)$ and sex for favor, (29.6\%). The common perpetrators of current sexual abuse were; casual partners, (57.1\%), teachers/lecturers/boss, (51.7\%) and intimate partner, $(33.0 \%)$. The commonest place of occurrence is the home of the perpetuator $(68.1 \%)$ followed by office, $(58.3 \%)$. Visiting of the perpetuator, (56\%) was the highest circumstances leading to abuse followed by violence/force, (47.3\%). The commonest consequence of abuse mentioned was emotional problem, (57.8\%) followed by psychological problems, (33.8\%) (Table 4).

Factors found to be significantly associated with life-time experience of sexual abuse were; tribe of respondent, $\left(\mathrm{X}^{2}=8.103, \mathrm{p}=0.004\right)$, type of secondary school attended, $\left(\mathrm{X}^{2}=10.432, \mathrm{p}=0.001\right)$, type of student in secondary school attended, $\left(\mathrm{X}^{2}=11.812, \mathrm{p}=0.001\right)$, faculty of study, $\left(\mathrm{X}^{2}=41.147, \mathrm{p}=0\right)$, level/year in school, $\left(X^{2}=7.321, p=0.007\right)$, persons shared room with, $\left(X^{2}=44.813, p=0\right)$, and level of knowledge about sexual abuse, $\left(\mathrm{X}^{2}=15.454, \mathrm{p}<0.001\right)$. Students who were non-Igbos, (OR: 2.38; 1.29 - 4.39), attended private secondary schools, (OR: 1.57; 1.08 - 2.29), were day students in secondary schools attended, (OR: 2.19; 1.21 - 3.97), currently studying in Faculty of Health Sciences, (OR: 12.19; $6.64-22.39$ ) and at 100 level of their study, (OR: $2.41 ; 1.25$ - 4.63) were more likely to have experienced sexual abuse in their life-time than others. On the other hand students who attended mission secondary schools, (OR: 0.59; 0.36 0.99), shared room currently with their boyfriend, (OR: 0.09; $0.04-0.20$ ) and 
Table 2. Awareness and knowledge of respondents about sexual abuse.

\begin{tabular}{|c|c|c|}
\hline VARIABLE & FREQUENCY & PERCENTAGE \\
\hline \multicolumn{3}{|l|}{ AWARE ABOUT SEXUAL ABUSE $(n=600)$} \\
\hline Yes & 491 & 81.8 \\
\hline No & 109 & 18.2 \\
\hline Total & 600 & 100 \\
\hline \multicolumn{3}{|l|}{${ }^{*}$ SOURCE OF INFORMATION $(n=491)$} \\
\hline Television & 300 & 61.1 \\
\hline Newspaper & 264 & 53.8 \\
\hline Friends & 260 & 53.0 \\
\hline Radio & 255 & 51.9 \\
\hline School & 232 & 47.3 \\
\hline Internet & 223 & 45.4 \\
\hline Parents/relatives & 179 & 36.5 \\
\hline Seminar/workshop & 161 & 32.8 \\
\hline Health personnel & 156 & 31.8 \\
\hline Books & 155 & 31.6 \\
\hline Church & 145 & 29.5 \\
\hline Market & 114 & 23.2 \\
\hline Billboards & 106 & 21.6 \\
\hline Government agencies & 94 & 19.1 \\
\hline Non-Governmental Organization & 89 & 18.1 \\
\hline \multicolumn{3}{|l|}{${ }^{\star *}$ MEANING OF SEXUAL ABUSE $(n=491)$} \\
\hline Sexual act without consent & 438 & 89.2 \\
\hline Rape & 287 & 58.5 \\
\hline Verbal excuse of sexual nature & 156 & 31.8 \\
\hline Sexual act by family member & 153 & 31.2 \\
\hline Sexual act for favor & 152 & 31.0 \\
\hline Sexual act by relatives/friends & 94 & 19.1 \\
\hline \multicolumn{3}{|l|}{${ }^{* *}$ FORMS OF SEXUAL ABUSE } \\
\hline \multicolumn{3}{|l|}{ KNOWN $(n=491)$} \\
\hline Fondling/Grabbling of sensitive body parts & 424 & 86.4 \\
\hline Rape & 328 & 66.8 \\
\hline Exposure to pornography & 228 & 46.4 \\
\hline Sex for favor & 154 & 31.4 \\
\hline Incest & 153 & 31.2 \\
\hline Degrading sexual comments & 138 & 28.1 \\
\hline Oral contact with genitalia & 135 & 27.5 \\
\hline Penetration with digits & 117 & 23.8 \\
\hline \multicolumn{3}{|l|}{${ }^{*}$ VICTIMS OF SEXUAL ABUSE $(n=491)$} \\
\hline Children & 364 & 74.1 \\
\hline Females & 336 & 69.0 \\
\hline Singles & 281 & 57.2 \\
\hline Married women & 214 & 43.6 \\
\hline Elderly women & 186 & 37.9 \\
\hline Males & 180 & 36.7 \\
\hline \multicolumn{3}{|l|}{ GENDER MOST AFFECTED $(n=600)$} \\
\hline Female & 578 & 96.3 \\
\hline Male & 22 & 3.7 \\
\hline Total & 600 & 100 \\
\hline
\end{tabular}




\section{Continued}

\begin{tabular}{|c|c|c|}
\hline \multicolumn{3}{|c|}{${ }^{* *}$ COMMON PERPETRATORS OF ABUSE $(n=491)$} \\
\hline Teachers/Lecturers & 355 & 72.3 \\
\hline Intimate partner & 217 & 44.2 \\
\hline Casual partners & 208 & 42.4 \\
\hline Family members/Friends & 192 & 39.1 \\
\hline Colleagues & 171 & 34.8 \\
\hline Spiritual leaders & 131 & 26.7 \\
\hline Parents & 127 & 25.9 \\
\hline \multicolumn{3}{|l|}{ COMMON PLACE OF ABUSE $(n=491)$} \\
\hline Secret corners & 349 & 71.1 \\
\hline School/office & 331 & 67.4 \\
\hline Clubs & 288 & 58.7 \\
\hline Home & 282 & 57.4 \\
\hline Hotels & 254 & 51.7 \\
\hline Public places & 211 & 43.0 \\
\hline \multicolumn{3}{|c|}{${ }^{*}$ FACTORS LEADING TO SEXUAL ABUSE $(n=491)$} \\
\hline Ignorance & 214 & 14.4 \\
\hline Financial problems & 196 & 13.2 \\
\hline Indecent dressing & 158 & 10.6 \\
\hline Poor family upbringing & 130 & 8.8 \\
\hline Lack of self-control & 128 & 8.6 \\
\hline Social problems & 128 & 8.3 \\
\hline Pornography & 116 & 7.8 \\
\hline Peer group pressure & 103 & 6.9 \\
\hline For favor done & 97 & 6.1 \\
\hline Love for pleasure & 80 & 5.4 \\
\hline Internet/social media & 75 & 5.1 \\
\hline Personal greed & 63 & 4.2 \\
\hline Others $^{1}$ & 25 & 1.7 \\
\hline \multicolumn{3}{|c|}{${ }^{*}$ HOW TO PREVENT OF SEXUAL ABUSE $(n=491)$} \\
\hline Self-control & 336 & 68.4 \\
\hline Avoid lonely visitations & 225 & 45.8 \\
\hline Punish offenders & 215 & 43.8 \\
\hline Avoid bad company & 185 & 37.7 \\
\hline Adequate security & 170 & 34.6 \\
\hline Strict regulations & 154 & 31.4 \\
\hline Use of law enforcements agents & 152 & 31.0 \\
\hline Empowerment of women & 130 & 26.5 \\
\hline Information dissemination & 122 & 24.9 \\
\hline Proper psychiatric examinations & 120 & 24.4 \\
\hline Proper monitoring of event & 106 & 21.6 \\
\hline \multicolumn{3}{|c|}{${ }^{* *}$ CONSEQUENCES MENTIONED $(\mathrm{n}=491)$} \\
\hline Sexually transmitted infections & 382 & 77.8 \\
\hline Emotional problems & 338 & 67.2 \\
\hline Psychological problems & 315 & 64.2 \\
\hline Physical injuries & 304 & 61.9 \\
\hline Social problems & 200 & 40.7 \\
\hline Morbidities & 176 & 35.8 \\
\hline Mortalities & 169 & 34.4 \\
\hline \multicolumn{3}{|l|}{ LEVEL OF KNOWLEDGE $(n=600)$} \\
\hline Poor $(0 \%-49 \%)$ & 282 & 47.0 \\
\hline Moderate $(50 \%-79 \%)$ & 243 & 40.5 \\
\hline Good $(\leq 80 \%)$ & 75 & 12.5 \\
\hline Total & 600 & 100 \\
\hline
\end{tabular}

${ }^{* *}=$ Multiple Response Applicable, Others ${ }^{1}=$ war/crisis, family problems. 
Table 3. Prevalence and pattern of sexual abuse.

\begin{tabular}{|c|c|c|}
\hline VARIABLE & FREQUENCY & PERCENTAGE \\
\hline \multicolumn{3}{|l|}{ EVER BEEN ABUSED $(n=600)$} \\
\hline Yes & 204 & 34.0 \\
\hline No & 396 & 66.0 \\
\hline Total & 600 & 100 \\
\hline \multicolumn{3}{|l|}{ FREQUENCY OF ABUSE $(\mathrm{n}=204)$} \\
\hline Once & 94 & 46.1 \\
\hline $2-4$ times & 69 & 33.8 \\
\hline Many times & 41 & 20.1 \\
\hline Total & 204 & 100 \\
\hline \multicolumn{3}{|l|}{${ }^{* *}$ FORMS OF SEXUAL ABUSE EXPERIENCED $(\mathrm{n}=204)$} \\
\hline Fondling/grabbing of sensitive body parts/kissing & 107 & 52.5 \\
\hline Rape & 39 & 19.1 \\
\hline Sex for favor & 27 & 13.2 \\
\hline Degrading sexual comments & 26 & 12.8 \\
\hline Anal intercourse & 17 & 8.3 \\
\hline Exposure to pornography & 14 & 6.8 \\
\hline Penetration with digits & 14 & 6.8 \\
\hline Incest & 12 & 5.8 \\
\hline Oral sex & 9 & 4.4 \\
\hline \multicolumn{3}{|l|}{${ }^{* *}$ PLACES OF OCCURRENCE $(n=204)$} \\
\hline Home of perpetrator & 148 & 72.6 \\
\hline Office & 54 & 26.5 \\
\hline Hostel & 33 & 16.2 \\
\hline Road & 32 & 15.7 \\
\hline Hotel & 31 & 15.2 \\
\hline Car & 23 & 11.3 \\
\hline Classroom & 21 & 10.3 \\
\hline Home of victim/Bush/party place & 7 & 3.4 \\
\hline \multicolumn{3}{|l|}{${ }^{*}$ CIRCUMSTANCES LEADING TO ABUSE $(\mathrm{n}=204)$} \\
\hline Visitation to home of perpetrator & 137 & 67.2 \\
\hline Violence/force & 71 & 34.8 \\
\hline Seeking favor & 44 & 21.6 \\
\hline Indecent dressing & 38 & 18.6 \\
\hline Drunkenness & 25 & 12.3 \\
\hline Drugs/Displacement/Crises & 6 & 2.9 \\
\hline \multicolumn{3}{|l|}{ CURRENT ABUSE IN LAST ONE YEAR $(n=600)$} \\
\hline Yes & 91 & 15.2 \\
\hline No & 509 & 84.8 \\
\hline Total & 600 & 100.0 \\
\hline \multicolumn{3}{|l|}{${ }^{* * F O R M}$ OF ABUSE CURRENTLY } \\
\hline \multicolumn{3}{|l|}{ EXPERIENCED $(n=91)$} \\
\hline Fondling/Grabbing of body parts/kissing & 56 & 61.5 \\
\hline Rape & 37 & 40.7 \\
\hline Sex for favor & 27 & 29.6 \\
\hline Degrading sexual comment & 26 & 28.6 \\
\hline Exposure to pornography/internet sex & 14 & 15.4 \\
\hline Incest & 12 & 13.2 \\
\hline Penetration with digits & 10 & 11.0 \\
\hline Oral sex & 9 & 9.9 \\
\hline
\end{tabular}




\section{Continued}

\begin{tabular}{|c|c|c|}
\hline \multicolumn{3}{|l|}{${ }^{*}$ MAIN PERPETRATORS $(\mathrm{n}=91)$} \\
\hline Casual partner & 52 & 57.1 \\
\hline Teacher/Lecturer/Boss & 47 & 51.7 \\
\hline Intimate partner & 30 & 33.0 \\
\hline Family member & 16 & 17.6 \\
\hline Friend & 14 & 15.4 \\
\hline Spiritual leader & 12 & 13.2 \\
\hline Colleague & 12 & 13.2 \\
\hline Stranger & 5 & 5.5 \\
\hline \multicolumn{3}{|l|}{${ }^{*}$ PLACES OF ABUSE $(n=91)$} \\
\hline Home of perpetrator & 63 & 69.2 \\
\hline Office & 53 & 58.2 \\
\hline Hostel & 31 & 36.3 \\
\hline Road & 22 & 24.2 \\
\hline Car & 18 & 19.8 \\
\hline Classroom & 14 & 15.4 \\
\hline Hotel & 12 & 13.2 \\
\hline Victims home/bush/party place & 3 & 3.3 \\
\hline \multicolumn{3}{|c|}{ 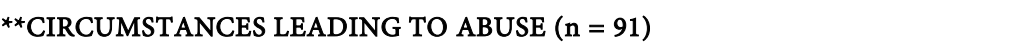 } \\
\hline Visitation & 51 & 56.0 \\
\hline Violence/force & 43 & 47.3 \\
\hline Seeking favor & 22 & 24.1 \\
\hline Indecent dressing & 22 & 24.1 \\
\hline Drunkenness & 19 & 9.9 \\
\hline Drugs/Displacement/Crises & 4 & 4.4 \\
\hline \multicolumn{3}{|c|}{${ }^{*}$ CONSEQUENCES OF THE ABUSE $(n=204)$} \\
\hline Emotional problems & 118 & 57.8 \\
\hline Psychological problems & 69 & 33.8 \\
\hline Had STI & 49 & 24.0 \\
\hline Physical injury & 38 & 18.6 \\
\hline Mental problems & 27 & 13.2 \\
\hline Social problems & 26 & 12.7 \\
\hline UTI/other morbidities & 16 & 7.8 \\
\hline Infertility & 8 & 3.9 \\
\hline
\end{tabular}

${ }^{* *}=$ Multiple response.

had good knowledge about sexual abuse, (OR: 0.24; $0.12-0.51$ ) were the least likely to have experienced sexual abuse in their life-time (Table 5).

Factors found to be significantly associated with current experience of sexual abuse among the students were; age of the respondents, $\left(X^{2}=6.630, p=0.010\right)$, tribe of respondents, $\left(X^{2}=7.120, p=0.008\right)$, marital status, $\left(X^{2}=14.290, p<\right.$ $0.001)$, type of student in secondary school attended, $\left(X^{2}=10.746, p=0.001\right)$, Faculty of study, $\left(X^{2}=38.005, p=0\right)$, level/year in school, $\left(X^{2}=22.205, p<\right.$ $0.0001)$, persons currently shared room with, $\left(X^{2}=19.520, p<0.0001\right)$, and level of knowledge about sexual abuse, $\left(\mathrm{X}^{2}=7.170, \mathrm{p}=0.007\right)$. Students who were non-Igbos, (OR: 2.48; 1.25 - 4.94), ever married, (OR: 2.79; 1.61 - 4.84), currently studying in Faculty of Health Sciences, (OR: 8.09; 4.60 - 14.22) and in 100 level of their study, (OR: $4.61 ; 2.32$ - 9.18) were more likely to have currently experienced sexual abuse than others. On the other hand students within the age 
Table 4. Determinants of life-time experience of sexual abuse among students.

\begin{tabular}{|c|c|c|c|c|c|}
\hline \multirow{2}{*}{ VARIABLES } & \multicolumn{3}{|c|}{ EVER EXPERIENCE OF SEXUAL ABUSE } & \multirow{2}{*}{$\begin{array}{c}\left.\text { STATISTICS/( } \mathrm{X}^{2}\right) \\
\text { P-VALUE }\end{array}$} & \multirow{2}{*}{ OR $(95 \% \mathrm{CI})$} \\
\hline & YES (\%) & NO (\%) & TOTAL (\%) & & \\
\hline \multicolumn{6}{|c|}{ AGE GROUP } \\
\hline$<20$ & $67(29.1)$ & $163(70.9)$ & $230(100)$ & \multirow{4}{*}{$\begin{array}{c}0.042 \\
\mathrm{p}=0.838\end{array}$} & \multirow{4}{*}{$\begin{array}{c}1.00 \\
1.37(0.96-2.00) \\
1.67(0.94-2.96)\end{array}$} \\
\hline $21-25$ & $111(36.3)$ & $195(63.7)$ & $306(100)$ & & \\
\hline$>26$ & $26(40.6)$ & $38(59.4)$ & $64(100)$ & & \\
\hline Total & $204(34.0)$ & $396(66.0)$ & $600(100)$ & & \\
\hline \multicolumn{6}{|l|}{ TRIBE } \\
\hline Igbo & $180(32.4)$ & $375(67.6)$ & $555(100)$ & \multirow{3}{*}{$\begin{array}{c}8.103 \\
p=0.004^{*}\end{array}$} & \multirow{3}{*}{$\begin{array}{c}1.00 \\
2.38^{*}(1.29-4.39)\end{array}$} \\
\hline Non-Igbo & $24(53.3)$ & $21(46.7)$ & $45(100)$ & & \\
\hline Total & $204(34.0)$ & $396(66.0)$ & $600(100)$ & & \\
\hline \multicolumn{6}{|l|}{ RELIGION } \\
\hline Catholic & $98(33.6)$ & $194(66.4)$ & $292(100)$ & \multirow{5}{*}{$\begin{array}{c}0.074 \\
\mathrm{p}=0.785\end{array}$} & \multirow{5}{*}{$\begin{array}{c}1.00 \\
1.04(0.67-1.62) \\
1.10(0.75-1.63) \\
\mathrm{Na}\end{array}$} \\
\hline Orthodox & $42(34.4)$ & $80(65.6)$ & $122(100)$ & & \\
\hline Pentecostal & $64(35.8)$ & $115(64.2)$ & $179(100)$ & & \\
\hline Others $^{1}$ & $0(0.0)$ & $7(100.0)$ & $7(100)$ & & \\
\hline Total & $204(34.0)$ & $396(66.0)$ & $600(100)$ & & \\
\hline \multicolumn{6}{|c|}{ MARITAL STATUS } \\
\hline Single & $177(33.9)$ & $345(66.1)$ & $522(100)$ & \multirow{3}{*}{$\begin{array}{c}0.015 \\
p=0.902\end{array}$} & \multirow{3}{*}{$\begin{array}{c}0.97(0.59-1.60) \\
1.00\end{array}$} \\
\hline Ever married & $27(34.6)$ & $51(65.4)$ & $78(100)$ & & \\
\hline Total & $204(34.0)$ & $396(66.0)$ & $600(100)$ & & \\
\hline \multicolumn{6}{|c|}{ TYPE OF SECONDARY SCHOOL ATTENDED } \\
\hline Public & $76(31.8)$ & $163(68.2)$ & $239(100)$ & \multirow{4}{*}{$\begin{array}{c}10.432 \\
p=0.001^{*}\end{array}$} & \multirow{4}{*}{$\begin{array}{c}1.00 \\
1.57^{*}(1.08-2.29) \\
0.59^{*}(0.36-0.99)\end{array}$} \\
\hline Private & $102(42.3)$ & $139(57.7)$ & $241(100)$ & & \\
\hline Missionary & $26(21.7)$ & $94(78.3)$ & $120(100)$ & & \\
\hline Total & $204(34.0)$ & $396(66.0)$ & $600(100)$ & & \\
\hline \multicolumn{6}{|c|}{ LOCATION OF SECONDARY SCHOOL ATTENDED } \\
\hline Urban & $129(32.3)$ & $271(67.7)$ & $400(100)$ & \multirow{3}{*}{$\begin{array}{c}1.638 \\
p=0.200\end{array}$} & \multirow{3}{*}{$\begin{array}{c}1.00 \\
1.26(0.88-1.80)\end{array}$} \\
\hline Rural & $75(37.5)$ & $125(62.5)$ & $200(100)$ & & \\
\hline Total & $204(34.0)$ & $396(66.0)$ & $600(100)$ & & \\
\hline & & M OF SECOI & Y SCHOOL A & NDED & \\
\hline Girls only & $86(35.2)$ & $158(64.8)$ & $244(100)$ & & 100 \\
\hline Mixed & $118(33.1)$ & $238(66.9)$ & $356(100)$ & $\begin{array}{c}0.285 \\
n=0.594\end{array}$ & $10(078-155)$ \\
\hline Total & $204(34.0)$ & $396(66.0)$ & $600(100)$ & $\mathrm{p}=0.594$ & $1.10(0 . / 8-1.55)$ \\
\hline & TYPE & ГUDENT IN & NDARY SCH & ATTENDED & \\
\hline Boarding & $63(35.8)$ & $113(42.9)$ & $176(100)$ & & 100 \\
\hline Day student & $108(29.7)$ & $256(44.0)$ & $364(100)$ & 11.812 & $\begin{array}{c}1.00 \\
0.76(0.52-112)\end{array}$ \\
\hline Mixed & $33(55.0)$ & $27(13.1)$ & $60(100)$ & $p=0.001$ & $0.76(0.52-1.12)$ \\
\hline Total & $204(34.0)$ & $396(66.0)$ & $600(100)$ & & $2.19^{*}(1.21-3.97)$ \\
\hline FACULTY & & & & & \\
\hline Arts/Education & $97(26.2)$ & $273(73.8)$ & $370(100)$ & & \\
\hline Social/Sciences & $42(28.0)$ & $108(72.0)$ & $150(100)$ & 41.147 & $\begin{array}{c}1.00 \\
10(072-167)\end{array}$ \\
\hline Health Sciences & $65(81.3)$ & $15(18.7)$ & $80(100)$ & $\mathrm{p}=0^{*}$ & $1.10(0.72-1.67)$ \\
\hline Total & $204(34.0)$ & $396(66.0)$ & $600(100)$ & & $12.19^{*}(6.64-22.39)$ \\
\hline LEVEL IN SCHOC & & & & & \\
\hline 100 & $21(53.8)$ & $18(46.2)$ & $39(100)$ & & $241 *(125-463)$ \\
\hline$\geq 200$ & $183(32.6)$ & $378(67.4)$ & $561(100)$ & $\begin{array}{l}1.321 \\
-0007 *\end{array}$ & $2.41^{*}(1.25-4.63)$ \\
\hline Total & $204(34.0)$ & $396(100)$ & $600(100)$ & $\mathrm{p}=0.007^{*}$ & 1.00 \\
\hline
\end{tabular}




\begin{tabular}{|c|c|c|c|c|c|}
\hline \multicolumn{6}{|c|}{ RESIDENCE IN SCHOOL } \\
\hline School hostel & $47(30.3)$ & $108(69.7)$ & $155(100)$ & \multirow{4}{*}{$\begin{array}{c}2.237 \\
p=0.135\end{array}$} & \multirow{4}{*}{$\begin{array}{c}1.00 \\
1.34(0.89-2.01) \\
1.05(0.63-1.76)\end{array}$} \\
\hline Off campus & $118(36.8)$ & $203(63.2)$ & $321(100)$ & & \\
\hline Family house & $39(31.5)$ & $85(68.5)$ & $124(100)$ & & \\
\hline Total & $204(34.0)$ & $396(66.0)$ & $600(100)$ & & \\
\hline \multicolumn{6}{|c|}{ SHARING ROOM } \\
\hline Yes & $169(35.0)$ & $314(65.0)$ & $483(100)$ & \multirow{3}{*}{$\begin{array}{c}1.081 \\
\mathrm{p}=298\end{array}$} & \multirow{3}{*}{$\begin{array}{c}1.00 \\
0.79(0.51-1.23)\end{array}$} \\
\hline No & $35(29.9)$ & $82(70.1)$ & $117(100)$ & & \\
\hline Total & $204(34.0)$ & $396(66.0)$ & $600(100)$ & & \\
\hline \multicolumn{6}{|c|}{ PERSON SHARED ROOM WITH $n=483$} \\
\hline Female friend & $111(47.4)$ & $123(52.6)$ & $234(100)$ & \multirow{4}{*}{$\begin{array}{l}44.813 \\
\mathrm{p}=0^{*}\end{array}$} & \multirow{4}{*}{$\begin{array}{c}1.00 \\
0.09^{*}(0.04-0.20) \\
0.54^{*}(0.36-0.83)\end{array}$} \\
\hline Boy friend & $7(7.4)$ & $87(92.6)$ & $94(100)$ & & \\
\hline Relative & $51(32.9)$ & $104(67.1)$ & $155(100)$ & & \\
\hline TOTAL & $169(35.0)$ & $314(65.0)$ & $483(100)$ & & \\
\hline \multicolumn{6}{|c|}{ SOLE SPONSOR IN SCHOOL } \\
\hline Self & $13(52.0)$ & $12(48.0)$ & $25(100)$ & \multirow{3}{*}{$\begin{array}{c}3.767 \\
p=0.052\end{array}$} & \multirow{3}{*}{$\begin{array}{c}1.00 \\
0.46(0.21-1.03)\end{array}$} \\
\hline Non-self & $191(33.2)$ & $384(66.8)$ & $575(100)$ & & \\
\hline Total & $204(34.0)$ & $396(66.0)$ & $600(100)$ & & \\
\hline \multicolumn{6}{|c|}{ LEVEL OF KNOWLEDGE } \\
\hline Poor & $101(35.8)$ & $181(64.2))$ & $282(100)$ & \multirow{4}{*}{$\begin{array}{c}15.454 \\
p<0.001\end{array}$} & \multirow{4}{*}{$\begin{array}{c}1.00 \\
1.13(0.79-1.61) \\
0.24^{*}(0.12-0.51)\end{array}$} \\
\hline Moderate & $94(38.7)$ & $149(61.3)$ & $243(100)$ & & \\
\hline Good & $9(12.0)$ & $66(88.0)$ & $75(100)$ & & \\
\hline Total & $204(34.0)$ & $396(66.0)$ & $600(100)$ & & \\
\hline
\end{tabular}

* = statistically significant.

group of 21 - 25 years of age, (OR: 0.44; 0.22 - 0.86) who were day students during their secondary school education, (OR: $0.43 ; 0.27-0.70)$, currently shared room with their boyfriend, (OR: $0.12 ; 0.04-0.38$ ) and had good knowledge about sexual abuse, (OR: $0.21 ; 0.06-0.71)$ were the least likely to have currently experienced sexual abuse in the last one year than their counterparts.

\section{Discussion}

The mean age of the respondents in this survey was $21.7 \pm 3.2$ years which was similar to mean ages found in others studies conducted among students in tertiary institutions in Nigeria [10] [12] [29] [30] [31] [33] [34]. This is expected in university setting where majority of them are within the age range of youth and young adults. A higher proportion of the students $(81.8 \%)$ were aware of sexual abuse; though a small proportion of them (12.5\%) had good knowledge about sexual abuse and the common sources of information were from the mass media, friends, school, internet, parents and relatives. This level of awareness was similar to that reported among female students in Malawi [45] but lower than figures reported from Osun State, Nigeria (97\%) [12] and from India, (88.1\%) [46]. The common sources of information were similar to what was reported in some of the reviewed studies [46] [47]. This high awareness and low level of knowledge found in this study is worrisome which puts to question the quality 
Table 5. Determinants of current sexual abuse among students.

\begin{tabular}{|c|c|c|c|c|c|}
\hline \multirow{2}{*}{ VARIABLES } & \multicolumn{3}{|c|}{ CURRENT EXPERIENCE OF SEXUAL ABUSE } & \multirow{2}{*}{$\begin{array}{c}\text { STATISTICS }\left(\mathrm{X}^{2}\right) \\
\text { P-VALUE }\end{array}$} & \multirow{2}{*}{ OR $(95 \% \mathrm{CI})$} \\
\hline & YES (\%) & NO (\%) & TOTAL (\%) & & \\
\hline \multicolumn{6}{|l|}{ AGE GROUP } \\
\hline$<20$ & $40(17.4)$ & $190(82.6)$ & $230(100)$ & \multirow{4}{*}{$\begin{array}{c}6.630 \\
p=0.010^{*}\end{array}$} & \multirow{4}{*}{$\begin{array}{c}0.69(0.35-1.35) \\
0.44^{*}(0.22-0.86) \\
1.00\end{array}$} \\
\hline $21-25$ & $36(11.8)$ & $270(88.2)$ & $306(100)$ & & \\
\hline$>26$ & $15(23.4)$ & $49(76.6)$ & $64(100)$ & & \\
\hline Total & $91(15.2)$ & $509(84.8)$ & $600(100)$ & & \\
\hline \multicolumn{6}{|l|}{ TRIBE } \\
\hline Igbo & $78(14.1)$ & $477(85.9)$ & $555(100)$ & \multirow{3}{*}{$\begin{array}{c}7.120 \\
p=0.008^{*}\end{array}$} & \multirow{3}{*}{$\begin{array}{c}1.00 \\
2.48^{*}(1.25-4.94)\end{array}$} \\
\hline Non-Igbo & $13(28.9)$ & $32(71.1)$ & $45(100)$ & & \\
\hline Total & $91(15.2)$ & $509(84.8)$ & $600(100)$ & & \\
\hline \multicolumn{6}{|l|}{ RELIGION } \\
\hline Catholic & $47(16.1)$ & $245(83.9)$ & $292(100)$ & \multirow{5}{*}{$\begin{array}{c}0.382 \\
\mathrm{p}=0.536\end{array}$} & \multirow{5}{*}{$\begin{array}{c}1.00 \\
1.02(0.58-1.81) \\
0.81(0.47-1.37) \\
0.0\end{array}$} \\
\hline Orthodox & $20(16.4)$ & $102(83.6)$ & $122(100)$ & & \\
\hline Pentecostal & $24(13.4)$ & $155(86.5)$ & $179(100)$ & & \\
\hline Islam & $0(0.0)$ & $7(100.0)$ & $7(100)$ & & \\
\hline Total & $91(15.2)$ & $509(84.8)$ & $600(100)$ & & \\
\hline \multicolumn{6}{|c|}{ MARITAL STATUS } \\
\hline Single & $68(13.0)$ & $454(87.0)$ & $522(100)$ & \multirow{3}{*}{$\begin{array}{c}14.290 \\
\mathrm{p}<0.001\end{array}$} & \multirow{3}{*}{$\begin{array}{c}1.00 \\
2.79 *(1.61-4.84)\end{array}$} \\
\hline Ever married & $23(29.5)$ & $55(70.5)$ & $78(100)$ & & \\
\hline Total & $91(15.2)$ & $509(84.8)$ & $600(100)$ & & \\
\hline \multicolumn{6}{|c|}{ TYPE OF SECONDARY SCHOOL ATTENDED } \\
\hline Public & $40(16.7)$ & $199(83.3)$ & $239(100)$ & \multirow{4}{*}{$\begin{array}{c}0.062 \\
p=0.804\end{array}$} & \multirow{4}{*}{$\begin{array}{c}1.00 \\
0.76(0.46-1.26) \\
0.94(0.52-1.70)\end{array}$} \\
\hline Private & $32(13.3)$ & $209(86.7)$ & $241(100)$ & & \\
\hline Missionary & $19(15.8)$ & $101(84.2)$ & $120(100)$ & & \\
\hline Total & $91(15.2)$ & $509(84.8)$ & $600(100)$ & & \\
\hline \multicolumn{6}{|c|}{ LOCATION OF SECONDARY SCHOOL ATTENDED } \\
\hline Urban & $54(13.5)$ & $346(86.5)$ & $400(100)$ & \multirow{3}{*}{$\begin{array}{c}2.591 \\
p=0.108\end{array}$} & \multirow{3}{*}{$\begin{array}{c}1.00 \\
1.45(0.92-2.30)\end{array}$} \\
\hline Rural & $37(18.5)$ & $163(81.5)$ & $200(100)$ & & \\
\hline Total & $91(15.2)$ & $509(84.8)$ & $600(100)$ & & \\
\hline & & ORM OF SE & ARY SCHOO & & \\
\hline Girls only & $44(18.0)$ & $200(82.0)$ & $244(100)$ & & \\
\hline Mixed school & $47(13.2)$ & $309(86.8)$ & $356(100)$ & 2.626 & 1.00 \\
\hline Total & $91(15.2)$ & $509(84.8)$ & $600(100)$ & $\mathrm{p}=0.105$ & $1.45(0.92-2.26)$ \\
\hline & TYPE & DENT IN SE & ARY SCHOO & TTENDED & \\
\hline Boarding & $39(22.2)$ & $137(77.8)$ & $176(100)$ & & \\
\hline Day student & $40(11.0)$ & $324(89.0)$ & $364(100)$ & 10.746 & 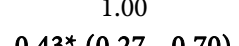 \\
\hline Mixed & $12(20.0)$ & $48(80.0)$ & $60(100)$ & $\mathrm{p}=0.001^{\star}$ & $0.43^{*}(0.27-0.70)$ \\
\hline Total & $91(15.2)$ & $509(84.8)$ & $600(100)$ & & $0.87(0.43-1.82)$ \\
\hline FACULTY & & & & & \\
\hline Art/Education & $34(9.2)$ & $336(90.8)$ & $370(100)$ & & \\
\hline Social/Sciences & $21(14.0)$ & $129(86.0)$ & $150(100)$ & 38.005 & 1.00 \\
\hline Health sciences & $36(45.0)$ & $44(55.0)$ & $80(100)$ & $\mathrm{p}=0^{*}$ & $\begin{array}{c}1.61(0.90-2.88) \\
80 *(460-1420)\end{array}$ \\
\hline Total & $91(15.2)$ & $509(84.8)$ & $600(100)$ & & $8.09 *(4.60-14.22)$ \\
\hline LEVEL IN SCHOC & & & & & \\
\hline 100 & $15(38.5)$ & $24(61.5)$ & $39(100)$ & & \\
\hline$\geq 200$ & $76(13.5)$ & $485(86.5)$ & $561(100)$ & 22.205 & $4.61^{*}(2.32-9.18)$ \\
\hline Total & $91(15.2)$ & $509(84.8)$ & $600(100)$ & $\mathrm{p}<0.0001$ & 1.00 \\
\hline
\end{tabular}




\begin{tabular}{|c|c|c|c|c|c|}
\hline \multicolumn{6}{|c|}{ RESIDENCE IN CURRENT SCHOOL } \\
\hline School hostel & $28(18.1)$ & $127(81.9)$ & $155(100)$ & \multirow{4}{*}{$\begin{array}{c}0.242 \\
p=0.623\end{array}$} & \multirow{4}{*}{$\begin{array}{c}1.00 \\
0.76(0.45-1.27) \\
0.72(0.37-1.39)\end{array}$} \\
\hline Off campus & $46(14.3)$ & $275(85.7)$ & $321(100)$ & & \\
\hline Family house & $17(13.7)$ & $107(86.3)$ & $124(100)$ & & \\
\hline Total & $91(15.2)$ & $509(84.8)$ & $600(100)$ & & \\
\hline \multicolumn{6}{|c|}{ SHARING ROOM } \\
\hline Yes & $75(15.5)$ & $408(84.5)$ & $483(100)$ & \multirow{3}{*}{$\begin{array}{c}0.251 \\
\mathrm{p}=0.616\end{array}$} & \multirow{3}{*}{$\begin{array}{c}1.00 \\
0.86(0.48-1.54)\end{array}$} \\
\hline No & $16(13.7)$ & $101(86.3)$ & $117(100)$ & & \\
\hline \multirow[t]{2}{*}{ Total } & $91(15.2)$ & $509(84.8)$ & $600(100)$ & & \\
\hline & \multicolumn{4}{|c|}{ PERSONS SHARED WITH $(n=483)$} & \\
\hline Female friend & $52(22.2)$ & $182(69.3)$ & $234(100)$ & \multirow{4}{*}{$\begin{array}{c}19.520 \\
p<0.0001\end{array}$} & \multirow{4}{*}{$\begin{array}{c}1.00 \\
0.12^{*}(0.04-0.38) \\
0.52^{*}(0.30-0.91)\end{array}$} \\
\hline Boyfriend & $3(3.2)$ & $91(96.8)$ & $94(100)$ & & \\
\hline Relative & $20(12.9)$ & $135(87.1)$ & $155(100)$ & & \\
\hline \multirow[t]{2}{*}{ Total } & $75(15.5)$ & $408(84.5)$ & $483(100)$ & & \\
\hline & & SOLE SPOI & N SCHOOL & \multirow{5}{*}{$\begin{array}{c}1.041 \\
\mathrm{p}=0.308\end{array}$} & \multirow{4}{*}{$\begin{array}{c}1.00 \\
2.11(0.49-9.09)\end{array}$} \\
\hline SELF & $2(8.0)$ & $23(92.0)$ & $25(100)$ & & \\
\hline Non-self & $89(15.4)$ & $486(84.5)$ & $575(100)$ & & \\
\hline \multirow[t]{2}{*}{ Total } & $91(15.2)$ & $509(84.8)$ & $600(100)$ & & \\
\hline & & LEVEL O & WLEDGE & & \\
\hline Poor & $46(16.3)$ & $236(83.7)$ & $282(100)$ & \multirow{4}{*}{$\begin{array}{c}7.170 \\
p=0.007^{*}\end{array}$} & \multirow{4}{*}{$\begin{array}{c}1.00 \\
1.07(0.68-1.70) \\
0.21^{*}(0.06-\mathbf{0 . 7 1})\end{array}$} \\
\hline Moderate & $42(17.2)$ & $201(82.7)$ & $243(100)$ & & \\
\hline Good & $3(4.0)$ & $72(96.0)$ & $75(100)$ & & \\
\hline Total & $91(15.2)$ & $509(84.8)$ & $600(100)$ & & \\
\hline
\end{tabular}

* = statistically significant.

of information they heard about sexual abuse and who was the person that taught them about it. The mass media which is the commonest source of information in this study will not be able to always give an in-depth information on most health related matters and therefore should not usually be a reliable source when it comes to appropriate knowledge about health issues. The prevalence of life-time experience of sexual abuse in this study was $34.0 \%$. This figure was within the range reported in WHO multi-county life-time prevalence of sexual partners violence among women aged 15 - 49 years, (6\% to 59\%) [16] [17]. It was also comparable to the prevalence rate reported in a university from the same region of the country, (36.7\%) [29] but studies from two other regions of the country; South-South region, (51.7\% and 46.7\%) [30] [31] and South West, (58\%) [48] reported higher prevalence rates. Nevertheless, lower figures were reported among students from universities in Northern region of the country, (13.8\% and 22.2\%) [33] [34]. This variation in life-time prevalence rates from different studies could be due to the differences that might have existed in the methodologies used, definition criteria applied, cultural and religious issues and level of awareness about sexual abuse. The prevalence of current sexual abuse in the last one year preceding study was $15.2 \%$. This was lower than the figure reported among female students in Ethiopia which stood at 24.4\% [41]. The commonest form of sexual abuse reported in this study was fondling/ grabbing of sensitive body parts which was followed by rape. This was similar to what was 
reported in a study among female university students in the south-south region of the country [31], and also from Malawi where they reported touching of breast in a sexual manner as the most common form [47], but differs with the report among female students from Calabar, Nigeria which reported unwanted sexual remarks and advances as the commonest form [30]. Majority of the perpetrators where men known to the victims which were mostly casual partners, teachers/lectures or intimate partners and this act occurs mostly during visitations at the home or office of the perpetrators. The study in Port-Harcourt, South-south, Nigeria [31], also revealed that most of the perpetrators were known to the victims but reported intimate partners as the commonest perpetrators while our study reported casual partners. Other studies also reported similar pattern with slight differences, with a study in Calabar, Nigeria [30] implicating lectures as one of the common perpetrators while the study in Kano, Nigeria, did not implicate lectures as major perpetrators.

Factors found to influence current occurrence of sexual abuse in this study were; age, tribe, marital status, type of student in secondary school attended, faculty of study, persons currently shared rooms with, year in school and level of knowledge about sexual abuse. Those within the ages of 21 - 25 years were least likely to have been currently abused when compared to others. A study among Ethiopia female students [41] reported a similar pattern which showed that students 20 - 24 years of age were about 2 times more likely to have been currently abused than their counterparts in other age group. Nevertheless on the contrary Ogbonnaya et al., reported higher prevalence of sexual assaults in students greater than 30 years of age [29]. Also a special report from the US Department of Justice revealed that females aged 18 - 24 had higher rates of rape and sexual assaults than females in other age groups [49]. This could be explained by the fact that at this age group many young adults engage in risky behaviours that could expose them to sexual violence and abuse. Married non-Igbo females students were more likely to have been currently abused than singles Igbo girls. This was similar to what was reported in Ebonyi [29] but differs with that reported from the North East Nigeria which showed that singles were more abused than their married counterparts [33]. Yet some other studies did not show any association between marital associated and occurrence of sexual abuse [38] [50]. This higher prevalence among the married as reported in this survey could have been contributed to by their partners who could be living together with them, irrespective of the challenges they face in school with other students. Female students in the Faculty of Health Sciences, who shared room with a female friend, lived in a boarding school during secondary education and being a 100 level student were more likely to have been currently abused than their counterparts. The study in Port-Harcourt [31] reported a similar higher prevalence among students in their first year of study though a study from the North Eastern part of the country showed no association [33]. This could be due to the fact that most students in their first year, are usually young and naive and can be usually deceived by the perpetrators with little or no resistance. Another study in 
Nigeria [37], also reported some important family determinants of sexual abuse which were; parents not living together, not living with parents, family type and current parents marital status, which is similar to finding in our study in which those who shared room with a female friend outside family care and those who lived in school hostels during their secondary school days were more likely to have been currently abused than others. This finding of protective role of the family is not new in this part of the world where family ideals are kept and transferred from generation to generation, and must be obeyed by all members especially the young people. Knowledge about sexual abuse was found to influence the occurrence of reported sexual abuse with those that have high knowledge about sexual abuse being more likely to have been currently abused than those with low knowledge. This was not consistent with what was reported in Malawi ${ }^{45}$ which showed that there was no association between knowledge and occurrence of sexual abuse. It is only those who have in-depth knowledge about sexual abuse that could ascertain when it occurred and what really constitutes an abuse. This could be the reason why female students in Health Science where more likely to report abuse in this study than others. The common health consequences of sexual abuse reported by those abused were emotional problems, psychological problems and transmission of sexually transmitted infections (STI) among others. This was similar to what was reported from other studies which were largely mental health problems like depression and guilty, physical injury and transmission of STI [30] [33].

\section{Conclusion}

In conclusion our study revealed a high prevalence of sexual abuse among the female students and a sizable proportion of them experienced rape. We found that younger age group, women in their first year of study, women with low knowledge about sexual abuse and those living with female friends outside family care were more likely to have been currently sexually abused. Thus there is need to institute preventive strategies targeted at those at risk of being abused and also to teach reproductive health issues in their general courses. Universities should establish youth friendly centers to tackle specialized reproductive health problems associated with the youths. There is need to implement already existing laws to help manage issues of sexual abuse in our universities and also develop new policies where necessary to curb this public health menace that is capable of affecting the full development of our women.

\section{Limitation}

The study information was self-reported and could be subject to some form of bias, thus caution should be taken in generalizing the findings of this study.

\section{Acknowledgements}

I want to acknowledge the medical students who collected data for us and thank all the female students who participated in the study. 


\section{Author's Contributions}

We hereby declare that all the authors contributed to this research.

\section{Conflicting Interest}

We hereby declare no conflicting interest.

\section{Source of Finding}

There was no external source of finding.

\section{References}

[1] United Nations (2006) Secretary-General's In-Depth Study on All Forms of Violence against Women. United Nations, New York.

[2] National Population Commission [Nigeria] and ICF Macro (2014) Nigeria Demographic and Health Survey 2013. National Population Commission and ICF Macro, Abuja.

[3] Krug, E.G., Dahlberg, L.L., Mercy, J.A., Zwi, A.B. and Lozano, R. (2002) World Report on Violence and Health. World Health Organization, Geneva.

[4] Berlin, F.S., et al. (2002) Peer Commentaries on Green and Schmidt. Archives of Sexual Behaviour, 31, 497-502.

[5] Messman-Moore, T.L. and Long, P.J. (2000) Child Abuse and Re-Victimization in the Form of Adult Sexual Abuse, Adult Physical Abuse and Adult Psychological Maltreatment. Journal of Interpersonal Violence, 15, 489-502. https://doi.org/10.1177/088626000015005003

[6] Tracy, K. and Cruise, T. (2017) Sexual Abuse of Children and Adolescents. National Association of School Psychologist. http://www.naspoline.org/educators/sexualabuse.pdf

[7] Hassan, M., Awosan, K.J., Panti, A.A., Nasir, S., Tunau, K., Umar, A.G., Shehu, C.E., Ukwu, A.E. and Sulaiman, B. (2016) Prevalence and Pattern of Sexual Assaults in UsmanuDanfodiyo University Teaching Hospital, Sokoto, Nigeria. Pan African Medical Journal, 24, 332. https://doi.org/10.11604/pamj.2016.24.332.9462

[8] Lalor, K. (2004) Child Abuse in Sub-Saharan Africa a Literature Review. Child Abuse \& Neglect, 28, 439-460. https://doi.org/10.1016/j.chiabu.2003.07.005

[9] Johnson, C.F. (2004) Child Sexual Abuse. The Lancet, 364, 462-470. https://doi.org/10.1016/S0140-6736(04)16771-8

[10] Olley, B.O. (2008) Child Sexual Abuse, Harmful Alcohol Use and Age as Determinants of Sexual Risk Behaviors among Freshmen in a Nigeria University. African Journal of Reproductive Health, 12, 75-88.

[11] Grossin, C., Sibille, I., Lorinde La Grandmaison, G., Banar, A., Brion, F. and Durigan, M. (2003) Analysis of 418 Cases of Sexual Assault. Forensic Science International, 131, 125-130. https://doi.org/10.1016/S0379-0738(02)00427-9

[12] Taiwo, M.O., Omole, O.C. and Omole, O.E. (2014) Sexual Harassment and Psychological Consequences among Students in Higher Education Institutions in Osun State, Nigeria. International Journal of Applied Psychology, 4, 13-18.

[13] Morley, L. and Kussier, K. (2009) Sex Grades and Power: Gender Violence in Africa Higher Education. Cambridge Journal of Education, 41, 101-105. https://doi.org/10.1080/0305764X.2010.549453 
[14] World Health Organization (1997) United Nations Organization Declaration on the Elimination of All Forms of Violence against Women. UN General Assembly 1993, New York.

[15] Schei, B., Sidenia, K., Lundvall, L. and Otherson, G. (2003) Adult of Sexual Assault: Acute Medical Response and Police Reporting among Women Consulting at Center for Victims of Sexual Assault. Acta Obstetricia et Gynecologica Scandinavica, 82, 750-755.

[16] Boh, S., et al. (2014) Violence against Women in Latin America and the Caribbean: A Comparative Analysis of Population-Based Data from 12 Countries. Pan American Health Organization, Washington DC.

[17] World Health Organization/Pan American Health Organization (2012) Understanding and Addressing Violence against Women. http://www.who.int/en/

[18] Jewkes, R. (2001) Relationship Dynamics and Adolescent Pregnancy in South Africa. Social Science and Medicine, 5, 733-744. https://doi.org/10.1016/S0277-9536(00)00177-5

[19] Matasha, E. (1998) Sexual and Reproductive Health among Primary and Secondary School Pupils in Mwanza Tanzania: Need for Intervention. Aids Care, 10, 571-582. https://doi.org/10.1080/09540129848433

[20] Buga, G.A., Amoko, D.H. and Ncayiyana, D.J. (1996) Sexual Behavior, Contraceptive Practice and Reproductive Health among School Adolescents in Rural Transkei. South African Medical Journal, 86, 523-527.

[21] Centers for Disease Control and Prevention (1991-2011) High School Youth Risk Behavior Survey Data.

[22] Krebs, C.P., Linguist, C.H., Warner, T.D., Fisher, B.S. and Martin, S.L. (2009) College Women Experiences with Physically Forced, Alcohol or Other Drug-Enabled and Drug-Facilitated Sexual Assault before and after Entering College. Journal of American College Health, 57, 639-647. https://doi.org/10.3200/JACH.57.6.639-649

[23] Menick, D.M. (2002) Sexual Abuse at Schools in Cameroon: Results of a Survey-Action Programme in Yaoundé. Medecine Tropicale, 62, 58-62.

[24] Alexander, Y. (2017) Prevalence of Gender Based Violence in Northern Sierra Leone. Technical Report Series, Centers for Accountability and the Rule of Law (CARL-SL). http://www.carl-sl.org

[25] Yohannes, O.Y. (2007) Prevalence and Factors Related to Gender Based Violence among Female Students of Higher Learning Institutions in Mekelle Town, Tigray, Northern Ethiopia. Postgraduate Thesis (MPH), Department of Community Health, Addis Ababa University, Addis Ababa, 23-45.

[26] Appiah, C.D. and Cusack, K. (1999) Violence against Women and Children in Ghana: Report of a National Study on Violence. Gender Studies and Human Rights Documentation Centre, Accra, 57-81.

[27] Shumba, A. (2001) Who Guards the Guards in Schools? A Study of Reported Cases of Child Abuse by Teachers in Zimbabwean Secondary Schools. Sex Education, 1, 77-86. https://doi.org/10.1080/14681810120041733

[28] Rivers, R. (2000) Shattered Hopes: Study of Sexual Abuse of Girls. UNICEF Botswana, Gaborone, 34-45.

[29] Ogbonnaya, L.U., Ogbonnaya, C.E. and Emma-Echiegu, N.B. (2011) Prevalence of Sexual Harassment/Victimization of Female Students in Ebonyi State University, South East, Nigeria. Journal of Community Medicine and Primary Health Care, 23, 55-67. 
[30] Effa, E.F., Okokon, E.O., Nwagbara, A.B. and Bello, S. (2017) Pattern, Determinants and Mental Consequences of Sexual Violence among Female Undergraduate Students in Calabar Southern, Nigeria.

[31] Mezie-Okoye, M.M.M. and Alamina, F.F. (2014) Sexual Violence among Female Undergraduates in a Tertiary Institution in Port Harcourt: Prevalence, Pattern, Determinants and Health Consequences. African Journal of Reproductive Health, 18, 79-85.

[32] Gharoro, E.P., Enabudoso, E.J. and Sodje, D.K. (2011) Non-Consensual Sex in Benin. Nigerian Journal of Clinical Practice, 14, 190-194. https://doi.org/10.4103/1119-3077.84013

[33] Kullima, A.A., Kawuwa, M.B., Audu, B.M., Mairiga, A.G. and Bukar, M. (2010) Sexual Assault against Female Nigerian Students. African Journal of Reproductive Health, 14, 189-193.

[34] Iliyasu, Z., Abubakar, I.S., Aliyu, M.H., Galadanci, H.S. and Salihu, H.M. (2011) Prevalence and Correlates of Gender Based Violence among Female University Students in Northern Nigeria. African Journal of Reproductive Health, 15, 111-119.

[35] Jewkes, R. and Abrahams, N. (2002) The Epidemiology of Rape and Sexual Coercion in South Africa: An Overview. Social Science \& Medicine, 55, 1231-1244. https://doi.org/10.1016/S0277-9536(01)00242-8

[36] Fisher, B.S., Cullen, F.T. and Turner, M.G. (2000) The Sexual Victimization of College Women. US Department of Justice, Washington DC. https://doi.org/10.1037/e377652004-001

[37] Nlewem, C. and Amodu, O.K. (2017) Family Characteristics and Structure as Determinants of Sexual Abuse among Female Secondary School Students in Nigeria: A Brief Report. Journal of Child Sexual Abuse, 26, 453-464. https://doi.org/10.1080/10538712.2017.1293202

[38] Adejimi, A.A., Sabageh, O.A. and Adedokun, O.P. (2016) Experiences and Disclosures of Sexual Assault among Nigerian Undergraduates in a Tertiary Institution. Violence and Gender, 3, 208-215. https://doi.org/10.1089/vio.2015.0035

[39] Kunnuji, M.O.N. and Esiet, A. (2013) Prevalence and Correlates of Sexual Abuse among Female Out-of-School Adolescents in Iwaya Community, Lagos State, Nigeria. African Journal of Reproductive Health, 19, 82-90.

[40] Diwe, K.C., Duru, C.B., Aguocha, C.M., Uwakwe, K.A., Merenu, I.A., Emerole, C.O. and Iwu, A.C. (2016) Prevalence, Pattern and Determinants of Intimate Partner Violence among Single Females in Tertiary Institutions in Imo State, Nigeria. International Journal of Research and Review, 3, 26-35.

[41] Adirew, Y.M. and Hagos, M.A. (2017) Sexual Violence against Female University Students in Ethiopia. BMC International Health and Human Rights, 17, 19. https://doi.org/10.1186/s12914-017-0127-1

[42] Heise, L., Elisberg, M. and Gottmoeller, M. (2002) A Global Overview of Gender-Based Violence. International Journal of Gynecology \& Obstetrics, 78, S5-S14. https://doi.org/10.1016/S0020-7292(02)00038-3

[43] Ullman, S.E. (1996) Do Social Reactions to Sexual Assault Victim Vary by Support Provider? Violence and Victim, 11, 143-157.

[44] Cass, A.S. (2007) Routine Activities and Sexual Assault: An Analysis of Individuals and School-Level Factors. Violence and Victim, 22, 350-355. https://doi.org/10.1891/088667007780842810

[45] Cochran, W. (1997) Sampling Techniques. 3rd Edition, John Wiley and Sons, New 
York.

[46] Cynthie, V.T. and Relton, A. (2014) Awareness on Sexual Abuse among Young Women. Indian Journal of Applied Research, 4, 181-183.

[47] Dzimadzi, R. and Klopper, H. (2007) Knowledge of Sexual Abuse amongst Female Students in Malawi. Curationis, 30, 23-30.

https://doi.org/10.4102/curationis.v30i3.1094

[48] Arulogun, O.S., Omotosho, I.K. and Titiloye, M.A. (2013) Experience of Sexual Harassment and Coping Strategies among Students of the School of Nursing of a Tertiary Hospital in South West, Nigeria. International Journal of Nursing and Midwifery, 5, 70-75. https://doi.org/10.5897/IJNM2013.0099

[49] Sinozich, S. and Langton, L. (2017) Rape and Sexual Assault Victimization among College-Age Females, 1995-2013. US Department of Justice.

[50] Odu, B.K. and Olusegun, G.F. (2012) Determinates of Sexual Coercion among University Female Students in South-West, Nigeria. Journal of Emerging Trends in Educational Research and Policy Studies, 3, 915-920. 


\section{Appendix}

\section{Questionnaire Design}

\section{Section A-Socio Demographic Characteristics of Respondents}

1) Age as at last birthday years

2) Marital Status (a) single [ ] (b) married [ ] (c) separated [ ] (d) divorced [ ] (e) widowed [ ] (f) living with partner [ ]

3) Type of secondary school attended (a) public/government school [ ] (b) private school [ ]

4) Location of secondary school attended (a) Urban area [ ] (b) Rural area [ ]

5) Form of secondary school attended (a) girls only school [ ] (b) mixed secondary school

6) Type of residence in secondary school attended (a) lived in the boarding school [ ] (b) was a day student [ ] (c) mixed both as a student [ ]

7) Faculty/department

8) Level in school (a) 100 level [ ] (b) 200 level [ ] (c) 300 level [ ] (d) 400 level [ ] (e) 500 level [ ] (f) 600 level [ ]

9) Place of residence (a) school hostel [ ] (b) off campus [ ] (c) living with family members [ ]

10a) Do you share room with anybody yes [ ] No [ ]

10b) If yes, who are you sharing g with (a) female friend [ ] (b) boyfriend [ ] (c) relative [ ] (d) Others please specify

11) Who is your sole sponsor in school? (a) Both parents [ ] (b) father only [ ] (c) mother only [ ] (d) relative/family member [ ] (e) self [ ] (f) community/scholarship[ ] (g) others please specify

12) Religion (a) catholic [ ] (b) orthodox (Anglican, Methodist, Baptist) [ ] (c) Pentecostal [ ] (d) Islam [ ] (e) others please specify

13) Tribe? (a) Igbo [ ] (b) Yoruba [ ] (c) Hausa [ ] (d) Others

Section B-Awareness and Knowledge of Respondents about Sexual Abuse

14a) Have you heard about sexual abuse (a) yes [ ] (b) No [ ]

$14 \mathrm{~b})$ If yes what is your source (multiple response applied) (a) radio [ ] (b) television [ ] (c) newspaper [ ] (d) school [ ] (e) friends [ ] (f) parents [ ] (g) health personnel [ ] (h) church [ ] (i) market [ ] (j) seminars/workshop [ ] (k) books [ ] (l) bill board [ ] (m) non-governmental organization [ ] (n) government agencies [ ] (o) internet [ ] (p) others please specify

15) What do you understand as sexual abuse? (a) Sexual act without consent [ ] (b) sexual act for favour [ ] (c) verbal abuse of sexual nature [ ] (d) sexual act by family member/relative [ ]

16) What are the forms of sexual abuse that you know? (Multiple response applied) (a) fondling/grabbing of sensitive body part/unwanted touching [ ] (b) sex for favour [ ] (c) incest [ ] (d) exposure to pornography [ ] (e) degrading sexual comments [ ] (f) oral contacts with genitalia [ ] (g) penetration with digits [ [ (h) rape [l\} others please specify 
17) Who can be a victim (multiple response applicable) (a) children [ ] (b) married [ ] (c) singles [ ] (d) elderly [ ]

18) What gender is commonly affected? (a)Male [ ] (b) female [ ]

19) Who are common perpetrators (multiple response applicable) (a) parents [ ] (b) teachers/lecturers [ ] (c) intimate partner [ ] (d) colleagues [ ] (e) casual partner [ ] (f) family / parents friends [ ] (g) spiritual leaders [ ] (h) others please specify

20) Where do you think sexual abuse occurs (multiple response applicable) (a) the home [ ] (b) the schools/offices [ ] (c) the public places [ ] (d) the hotels [ ] the clubs (e) the secret corners [ ] (f) others please specify

21) What are the consequences of sexual abuse (multiple response applicable) (a) physical injuries [ ] (b) a sexually transmitted infection [ ] (c) emotional problems [ ] (d) infertility [ ] (e) psychological problem [ ] (f) social problem/stigma [ ] (g) morbidities [ ] (h) dissociation [ ] (l) mortality [ ] (j) others please specify

\section{Section C-Prevalence and Pattern of Sexual Abuse Experienced}

22a) Have you ever been sexually abused before? (a) Yes [ ] (b) No [ ]

22b) If yes, how many times? (a) Once [ ] (b) twice [ ] (c) three times [ ] (d) four times [ ] (e) Many [ ]

23) How were you abused?

24) Where did it occur? (Multiple response applicable) (a) Home of perpetrator [ ] (b) home of office [ ] (c) office [ ] (d) classroom [ ] (e) hostel [ ] (f) road [ ] (g) in a car [ ] (h) hotel [ ] (l) others please specify.

25) What circumstances led to the event (multiple response applicable) (a) Violence/force [ ] (b) visiting [ ] (c) drunk [ ] (d) seeking favour [ ] (e) indecent dressing [ ] (f) other please specify.

26a) Have you been sexually abused in the last one year? (a)Yes [ ] (b) No [ ]

26b) If yes, what form of sexual abuse have you had? (Multiple response applicable) (a) fondling/grabbing of sensitive body part/unwanted touching (b) vaginal/anal intercourse [ ] (c) sex for favour [ ] (d) incest [ ] (e) exposure to pornography [ ] (f) degrading sexual comments [ ] (g) oral contact with genitalia [ ] (h) penetration with digits [ ] (i) rape [ ] (j) other please specify.

27) Who were the persons that sexually abused you (Multiple response applicable) (a) My parents [ ] (b) teachers/lecturers/boss [ ] (c) intimate partner [ ] (d) colleagues [ ] (e) casual partner [ ] (f) family/parent's friends [ ] (g) Spiritual leaders [ ] (h) others please specify.....

28) Place the abused took placed? a) Home of perpetrator [ ] (b) home of office [ ] (c) office [ ] (d) classroom [ ] (e) hostel [ ] (f) road [ ] (g) in a car [ ] (h) hotel [ ] (1) others please specify.

29) Circumstances of the abuse (Multiple response applicable) (a) Violence/force [ ] (b) visiting [ ] (c) drunk [ ] (d) seeking favour [ ] (e) indecent dressing [ ] (f) other please specify....

30) Problems that the abuse cause you (multiple response applicable) (a) emo- 
tional [ ] (b) physical injury [ ] (c) transmission STI [ ] (d) mental problems [ ] (e) psychological problem [ ] (f) Social problem (g) morbidities [ ] (h) other please specify....... 